\title{
Pyrolysis-mass spectrometry (Py-MS) for the rapid epidemiological typing of clinically significant bacterial pathogens
}

\author{
R. FREEMAN, M. GOODFELLOW, F.K. GOULD*, S. J. HUDSON† and N. F. LIGHTFOOT‡
}

\begin{abstract}
Department of Microbiology, University of Newcastle upon Tyne, "Freeman Hospital, Newcastle upon Tyne, $\dagger$ Queen Elizabeth Hospital, Gateshead, and $\ddagger$ Regional Public Health Laboratory, Newcastle upon Tyne
\end{abstract}

\begin{abstract}
Summary. Fresh clinical isolates of Salmonella spp. and Streptococcus pyogenes were analysed by pyrolysis-mass spectrometry (Py-MS). The results formed the basis of mathematically derived characterisations of individual strains and these were compared with the results of phage typing for the salmonellas and $\mathbf{M}$ protein typing for the streptococci. Py-MS was shown to be a rapid and reproducible method for inter-strain comparisons, giving evidence of identity and non-identity between strains that agreed well with the results of conventional tests. Py-MS has potential value as a rapid, relatively inexpensive and highly discriminatory method of epidemiological analysis in bacterial disease.
\end{abstract}

\section{Introduction}

Pyrolysis of micro-organisms yields complex mixtures of products that can be analysed quantitatively by mass spectrometry. The results can be used in mathematically based characterisation techniques that lead to "fingerprinting" of the micro-organisms. ${ }^{1}$ The method can be used for identification, classification and typing of different microbes and offers evidence of identity and nonidentity with a high degree of resolution and speed. ${ }^{2}$

Previous reports have included results of studies on Listeria monocytogenes, ${ }^{3}$ Klebsiella spp.,${ }^{4}$ Bacillus thuringiensis, ${ }^{5}$ B. cereus ${ }^{6}$ and Candida albicans. ${ }^{7}$ The potential application of a single method to a range of different micro-organisms conventionally classified and typed by a variety of techniques such as serology, biochemistry, bacteriophage susceptibility and others, and the possibility of its application to species for which there are no current typing schęmes, makes pyrolysis-mass spectrometry (PyMS) attractive for the investigation of hospital cross-infection and community outbreaks of microbial diseases.

The pilot study described here was designed to test the usefulness of Py-MS for rapid microbial epidemiological investigations with fresh clinical isolates of two important but distinct human pathogens.

Received 6 Dec. 1989; accepted 16 Feb. 1990

\section{Materials and methods}

\section{Bacterial strains and media}

Isolates of Salmonella spp. and Streptococcus pyogenes were collected from routine clinical specimens submitted to Queen Elizabeth Hospital, Gateshead over a period of several weeks. Salmonellas (eight strains) were faecal isolates from cases of community-acquired diarrhoea and were stored on Nutrient-agar slopes (Lab M) before analysis. Eight strains of streptococci were isolated from throat swabs, in five instances taken from different patients involved in an outbreak of pharyngitis in a residential institution. They were stored in cryopreservative solution at $-20^{\circ} \mathrm{C}$ (Protect Bacterial Preservers; Technical Service Consultants Ltd) until required. Before analysis salmonellas were cultured on Columbia Agar (Lab M) and streptococci on Columbia Blood Agar (Lab $M)$. In each instance the subculture media used were of a single prepared batch. All subcultures were incubated at $37^{\circ} \mathrm{C}$ for $24 \mathrm{~h}$. Immediately before analysis one investigator coded and labelled the samples $\mathrm{S} 1-8$ for the salmonellas and A-H for the streptococci. Py-MS analysis was performed by the other investigators who were unaware of the identity of the individual isolates until after the completion of the analysis. Details of the strains examined, including the results of conventional typing are shown in the table.

\section{Sample preparation and Py-MS analysis}

Pyrolysis foils (Horizon Instruments) were used. A well-separated colony of the organism to be tested was identified and sampled with a flamed straight wire. Care 
was taken to avoid contamination with the medium. The material was smeared on to the foil to give a uniform surface coating and the foil was inserted into a pyrolysis tube (Horizon Instruments). The assembled tubes were heated at $80^{\circ} \mathrm{C}$ for $5 \mathrm{~min}$ in a hot air oven. Each sample was prepared in triplicate.

Sample tubes were processed in batches of 24 , i.e., the Salmonella samples and the Streptococcus samples in separate batches, on a Horizon Instruments PYMS 200X pyrolysis mass spectrometer. The time required for the analysis of each individual sample was approximately 2 min and the pyrolysis was at a Curie point of $530^{\circ} \mathrm{C}$ for a period of $4 \mathrm{~s}$. Products were ionised by collision with a crossing beam of low energy $(25 \mathrm{eV})$ electrons, and the ions were separated in a quadrupole mass spectrometer which scanned the pyrolysate 160 times at intervals of $0.35 \mathrm{~s}$ from the start of pyrolysis. Integration ion counts at unit mass intervals from 11 to 200 were recorded on floppy disk, together with the pyrolysis sequence number and total ion count for each individual sample.

\section{Data analysis}

Discriminant analysis was performed on normalised ion counts of the analysis set, with the replicate spectrum data for each strain labelled as a separate group. This analysis optimised the discrimination between strains, corrected for systematic intra-strain variation and covariance effects, and represented this optimised discrimination as co-ordinates on each orthogonal axis. The spectral data were presented in the form of an ordination diagram. The apparatus, technique and principles involved have been described previously. ${ }^{8}$

\section{Results}

The ordination diagram of the spectral data for the first two canonical discriminant axes, which account for $89.7 \%$ of inter-strain discrimination for the salmonellas is shown in fig. 1. Two strains ( $\mathrm{S} 1$ and S6) are obvious outliers whereas the remaining strains form a relatively close cluster at this low level of resolution. Strains $S 1$ and $S 6$ are $S$. typhimurium and $S$. panama respectively, whereas the remainder of the strains are $S$. enteritidis (table). The same data, with the two outliers, S1 and S6, edited out and thus relating to the $S$. enteritidis strains alone, are shown in fig. 2. The spectral data presented account for $82.6 \%$ of the inter-strain discrimination for Salmonella isolates S2, S3, S4, S5, S7 and S8. It can be seen that four strains (S25) are clustered but that $S 7$ and $S 8$ are outliers. Strain $S 8$ is $S$. enteritidis phage type 24, whereas strains S2-5 are all of phage type 4; strain S7 is also of phage type 4 but is tetracycline sensitive, whereas the clustered phage type 4 strains are resistant to tetracycline (table).
The ordination diagram of the spectral data for the first two canonical discriminant axes, which account for $73.4 \%$ of the inter-strain discrimination in the Str. pyogenes isolates, is shown in fig. 3. Three outliers are seen. Strains $\mathrm{C}$ and $\mathrm{F}$ are Str. pyogenes of $M$ types 1 and 12, respectively, and strain $G$ is M-untypable but typed as T6. The remaining strains (A, B, D, E and $\mathrm{H}$ ), which form a tight cluster, are type $\mathrm{M} 49$.

\section{Discussion}

This study was concerned with a small number of isolates of only two bacterial species. It was designed to test the hypothesis that Py-MS can be applied in the rapid typing of fresh clinical isolates. Under these circumstances, evidence of identity and non-identity between individual strains is the goal and in these terms the results are very encouraging. Identification of salmonellas to the species level is a task well within the compass of most routine laboratories but differentiation of strains within a species usually requires the services of a Reference Laboratory able to undertake phage typing. The Py-MS technique differentiated be-

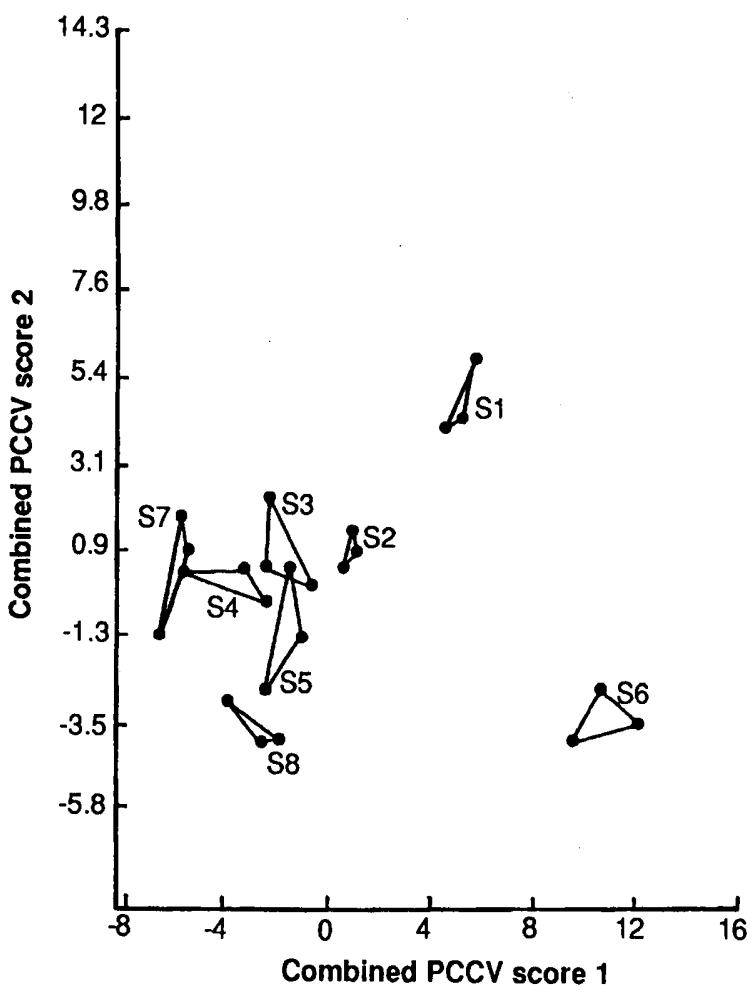

Fig. 1. Ordination diagram of spectral data for Salmonella strains S1-S8. The axes represent the first two canonical discriminant functions. Results of triplicate analysis on the same strain have been joined together. 
Table. Results of conventional typing of strains of Salmonella spp. and of Str. pyogenes

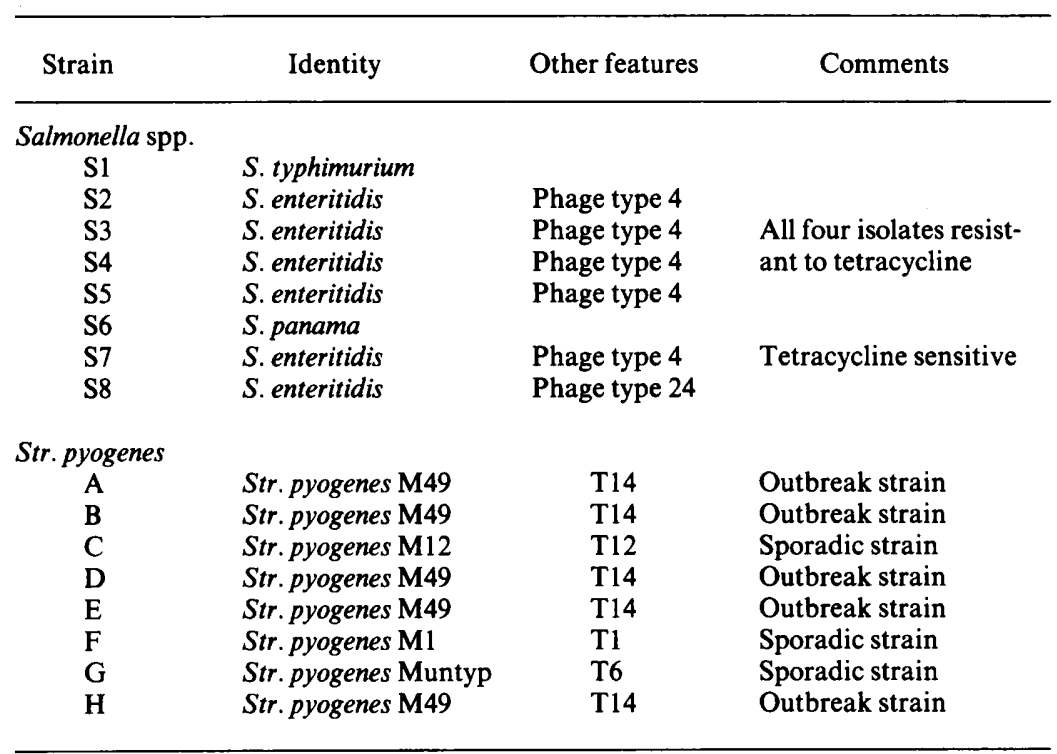

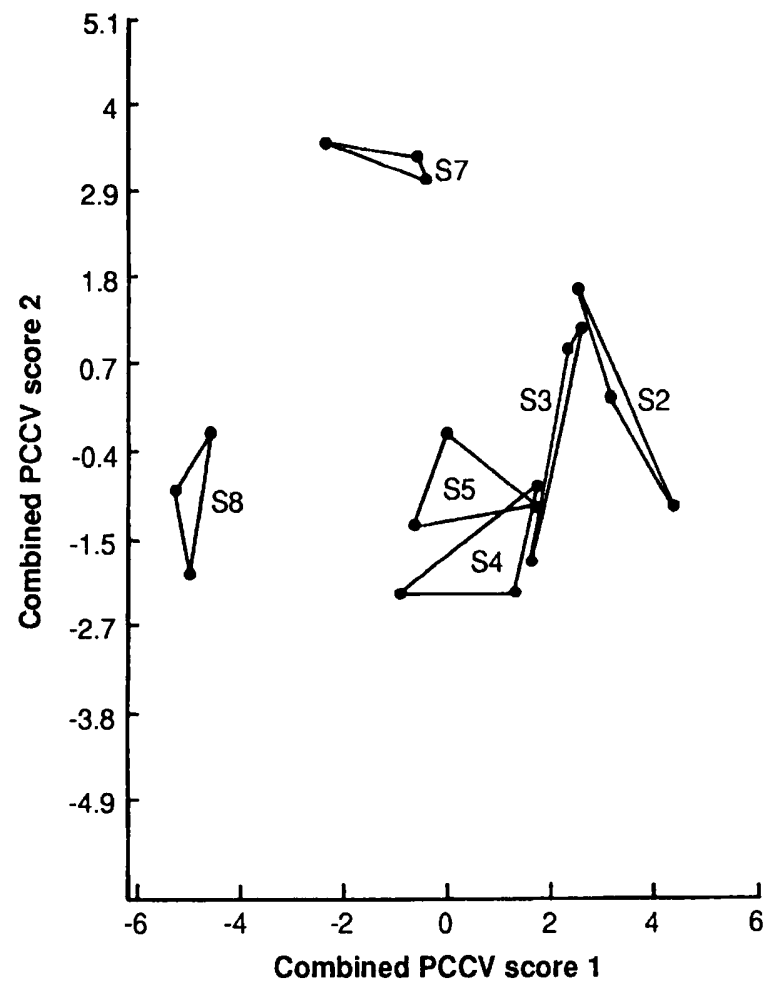

Fig. 2. Ordination diagram of spectral data for Salmonella strains S2, S3, S4, S5, S7 and S8. The axes represent the first two canonical discriminant functions. Results of triplicate analysis on the same strain have been joined together.

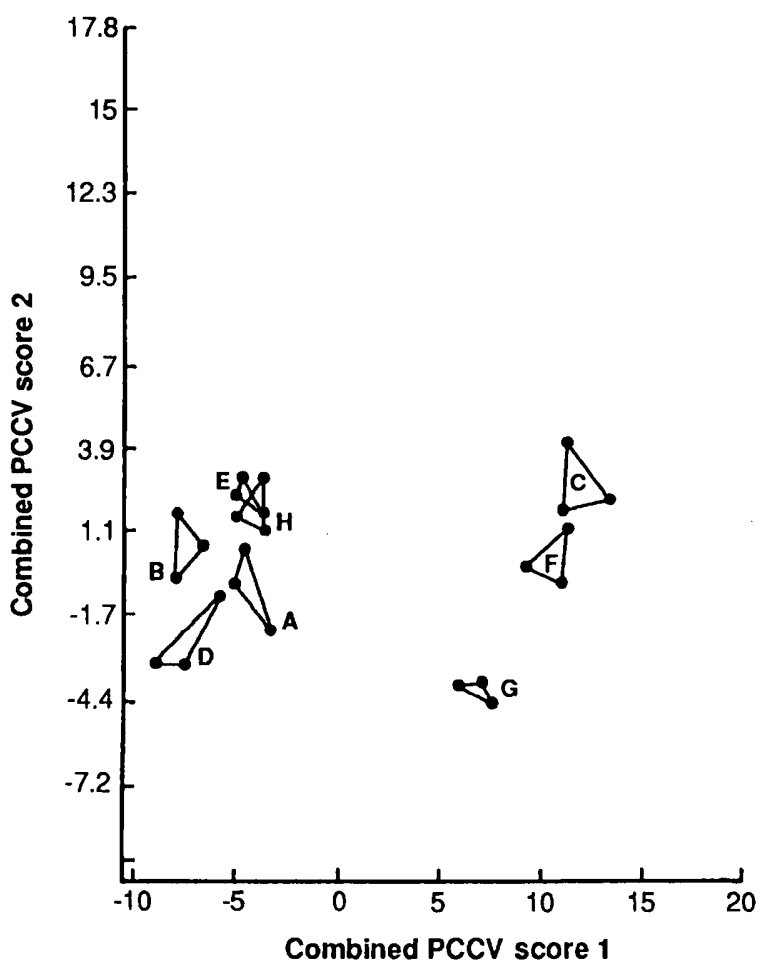

Fig. 3. Ordination diagram of spectral data for Str. pyogenes strains $\mathbf{A}-\mathbf{H}$. The axes represent the first two canonical discriminant functions. Results of triplicate analysis on the same strain have been joined together. 
tween different phage types of $S$. enteritidis and also suggested that one strain of phage type 4 was different from the remainder. Antibiotic sensitivity testing showed that this isolate was sensitive to tetracycline whereas the remaining four strains were resistant. Tetracycline resistance is relatively unusual in $S$. enteritidis phage type 4 in the UK, where most isolates are sensitive to all antibiotics. The four tetracycline-resistant strains were collected from a geographically restricted area during a short period of time and may well represent an unsuspected outbreak strain. It is also interesting to note a recent report ${ }^{9}$ which suggests that discrimination between strains of $S$. enteritidis phage type 4 can be achieved by plasmid profile analysis. The results presented here may be evidence of the same phenomenon.

The results with the strains of Str. pyogenes are equally interesting. This is another common human pathogen easily identified to species level but its typing requires specialised facilities and expertise. Py-MS separated the different $M$ types and clustered those of the same $M$ type, providing evidence of identity and non-identity which correlated with the results of conventional typing methods. The known outbreak strain was correctly distinguished from the sporadic isolates.

Py-MS analysis allowed the consecutive examination of isolates of two unrelated species on the same equipment within hours of each other. The results, in terms of identity and non-identity of the strains analysed, were clinically relevant and would have allowed confirmation or otherwise of an

\section{REFERENCES}

1. Gutteridge CS, Norris JR. The application of pyrolysis techniques to the identification of micro-organisms. $J$ Appl Bacteriol 1979; 47: 5-43.

2. Shute LA, Berkeley RCW, Norris JR, Gutteridge CS. Pyrolysis mass-spectrometry in bacterial systematics. In: Goodfellow M, Minnikin DE (eds) Chemical methods in bacterial systematics: London, Academic Press. 1985: 95-114.

3. Eshuis W, Kistemaker PG, Meuzelaar HLC. Some numerical aspects of reproducibility and specificity. In: Jones ER, Cramers CA (eds) Analytical pyrolysis: Amsterdam, Elsevier. 1977: 151-166.

4. Meuzelaar HL, Haverkamp J. Pyrolysis mass spectrometry of recent and fossil biomaterials. (Techniques and instrumentation in analytical chemistry 3) 1982 . Elsevier, Amsterdam.

5. Gutteridge CS, Vallis L, MacFie HJH. Numerical methods in the classification of micro-organisms by pyrolysis outbreak strain and would have facilitated appropriate action. Equivalent results by conventional methods for either of the species examined would require the services of distant Reference Laboratories before useful information could become available. The speed, both of the individual analyses and of the "on site" availability of a common method of typing markedly different species, makes Py-MS a very attractive technique for microbial epidemiology.

Py-MS is also likely to be comparatively inexpensive in comparison to conventional typing systems. Apart from the initial capital outlay, of approximately $£ 70000$, the consumable costs are low in comparison with the costs of serology and phage typing and have been estimated to be significantly less than $£ 1.00$ per sample. ${ }^{10}$ The method requires minimal labour input.

The present study does not allow strain identification but merely comparison of one strain with another. However, the technique has the capacity to retain data and compile a software "library". Use of reference strains and a detailed systematic analysis of accumulated data on a standardised basis should allow the development of a taxonomic database capable of adding final identification to inter-strain comparisons. The technique also has the attraction of offering a novel approach to the typing of as yet untypable species.

We are extremely grateful for the help, advice and enthusiastic co-operation of Horizon Instruments Ltd, Heathfield, Kent, in this study.

mass-spectrometry. In : Goodfellow M, Jones D, Priest FG (eds) Computer-assisted bacterial systematics. London, Academic Press. 1985: 369-401.

6. Shute LA, Gutteridge CS, Berkeley RCW. Pyrolysis mass spectrometry: a discriminatory technique with potential for rapid characterisation and identification of micro-organisms. XIV International Congress of Microbiology, 1986, Manchester; Abstract.

7. Magee JT, Hindmarch JM, Duerden BI, MacKenzie DWR. Pyrolysis mass spectrometry as a method for interstrain discrimination of Candida albicans. J Gen Microbiol 1988; 134: 2841-2847.

8. Aries RE, Gutteridge CS, Ottley TW. Evaluation of a lowcost, automated pyrolysis mass-spectrometer. $J$ Anal Appl Pyrolysis 1986; 9: 81-98.

9. Threlfall EJ, Rowe B, Ward LR. Subdivision of Salmonella enteritidis phage types by plasmid profile typing. Epidemiol Infect 1989; 102: 459-465.

10. Magee JT, Hindmarch JM, Bennett $\mathrm{KW}$, Duerden BI, Aries RE. A pyrolysis mass spectrometry study of fusobacteria. J Med Microbiol 1989; 28 : 227-236. 